\title{
Analysis of ESR1 and PIK3CA mutations in plasma cell-free DNA from ER-positive breast cancer patients
}

\author{
Takashi Takeshita ${ }^{1}$, Yutaka Yamamoto ${ }^{1}$, Mutsuko Yamamoto-Ibusuki², Mai \\ Tomiguchi ${ }^{1}$, Aiko Sueta ${ }^{1}$, Keiichi Murakami ${ }^{1}$, Yoko Omoto ${ }^{1,3}$ and Hirotaka Iwase ${ }^{1}$ \\ ${ }^{1}$ Department of Breast and Endocrine Surgery, Graduate School of Medical Science, Kumamoto University, Honjo, Chuo-Ku, \\ Kumamoto, Japan \\ ${ }^{2}$ Department of Molecular-Targeting Therapy for Breast Cancer, Kumamoto University Hospital, Honjo, Chuo-Ku, Kumamoto, \\ Japan \\ ${ }^{3}$ Department of Endocrinological and Breast Surgery, Graduate School of Medical Science, Kyoto Prefectural University of \\ Medicine, Hirokoji Agaru, Kawaramachi-Dori, Kamigyo-Ku, Kyoto, Japan \\ Correspondence to: Hirotaka Iwase, email: hiwase@kumamoto-u.ac.jp \\ Keywords: estrogen receptor-positive metastatic breast cancer, acquired endocrine therapy resistance, cell-free DNA, ESR 1 mu- \\ tations, PIK3CA mutations \\ Received: April 27, 2017 \\ Accepted: May 23, 2017 \\ Published: June 14, 2017
}

Copyright: Takeshita et al. This is an open-access article distributed under the terms of the Creative Commons Attribution License 3.0 (CC BY 3.0), which permits unrestricted use, distribution, and reproduction in any medium, provided the original author and source are credited.

\section{ABSTRACT}

Background: The measurement of ESR1 and PIK3CA mutations in plasma cellfree DNA (cfDNA) has been studied as a non-invasive method to quickly assess and monitor endocrine therapy (ET) resistant metastatic breast cancer (MBC) patients.

Methods: The subjects of this retrospective study were a total of 185 plasma samples from 86 estrogen receptor-positive BC patients, of which 151 plasma samples were from 69 MBC patients and 34 plasma samples were from 17 primary BC (PBC) patients. We developed multiplex droplet digital PCR assays to verify the clinical significance of ESR1 and PIK3CA mutations both in a snapshot and serially in these patients.

Results: cfDNA ESR1 and PIK3CA mutations were found in $28.9 \%$ and $24.6 \%$ of MBC patients, respectively. The relation between ESR1 or PIK3CA mutations and clinical features showed that ESR1 mutations occurred mostly in patients previously treated by ET, which was not the case for PIK3CA mutations. The analysis of the clinical impact of those mutations on subsequent lines of treatment for the 69 MBC patients revealed that both ESR1 and PIK3CA mutations detection were related to a shorter duration of ET effectiveness in univariate analysis but only for ESR1 mutations in multivariate analysis. The monitoring of cfDNA in a subset of 52 patients showed that loss of ESR1 mutations was related to a longer duration of response, which was not the case for PIK3CA mutations.

Conclusions: We have demonstrated the clinical significance of on-treatment ESR1 mutations both in a snapshot and serially in comparison with PIK3CA mutations.

\section{INTRODUCTION}

Approximately $80 \%$ of breast cancers (BCs) express the estrogen receptor alpha $(\mathrm{ER} \alpha)$, encoded by the ESRI gene, and endocrine therapy (ET) with selective ER modulators (SERMs) or aromatase inhibitors (AIs) is the mainstay of treatment for this group of patients because of their effectiveness balanced against their side effects. However, ET resistance occasionally occurs during the treatment of early $\mathrm{BC}$ and inevitably results in metastatic $\mathrm{BC}$ (MBC) [1]. ESR1 ligand binding domain (LBD) mutations constitutively activate the ER in a ligandindependent fashion [2-4] and they have attracted attention as a mechanism of ET resistance in MBC. These mutations were originally reported almost two decades ago [5$8]$, and recent large-scale next-generation sequencing (NGS) revealed that ESR1 mutations are present in approximately $20-50 \%$ of metastatic tissue samples treated 
with endocrine agents while these variants are absent or only present at very low frequencies in primary tumor samples [2-4]. These features indicate that the presence of ESR 1 mutations should be assessed in metastatic lesions. Circulating cell-free DNA (cfDNA) has been proposed to carry a comprehensive picture of metastatic tumor cells and genomic analysis of plasma cfDNA has been realized as a non-invasive method to quickly assess the mutational profiles and monitor molecular changes under treatment, using recent developments in digital genomic technologies [9]. Therefore, if ESR1 mutation status in cfDNA is predictive of response to ET, monitoring of this marker could be a useful method of informing treatment plans for subsequent metastatic disease.

$P I K 3 C A$ is an oncogene that encodes the $\mathrm{p} 110 \alpha$ component of phosphatidylinositol 3-kinase (PI3K) and $P I K 3 C A$ is a representative frequently-mutated gene, whose frequencies are $20 \%$ to $40 \%$ of all BCs $[10,11]$. Recently, in phase III randomized trials, the clinical significance of ESR1 mutations have been reported in the comparison with $P I K 3 C A$ mutations. In alteration frequency in metastatic versus primary tumors in the BOLERO-2 cohort, Hortobagyi et al. demonstrated that PIK3CA mutations had the highest frequency in $\mathrm{PBCs}$ and $\mathrm{MBCs}$ and that ESR1 mutations had higher frequency in MBCs than in PBCs [12]. More recently, in the BOLERO-2 study, Chandarlapaty and colleagues found that $28.8 \%(155 / 541)$ of ER-positive MBC patients had ESR1 mutations in plasma cfDNA [13] and 43.3\% (238/550) of ER-positive MBC patients had PIK3CA mutations in plasma cfDNA [14]. They also demonstrated that the difference of clinical features between ESR1 and PIK3CA mutations, namely, progression free survival (PFS) benefit of mammalian target of rapamycin (mTOR) inhibitor everolimus was maintained irrespective of PIK3CA mutations, but that was decreased according to the presence of ESR1 mutations [13, 14]. In another two phase III randomized trials, Fribbens and colleagues assessed ESR 1 mutations in cfDNA using digital PCR (dPCR) [15]. ESR 1 mutations were found in the plasma of $39.1 \%$ of patients $(63 / 161)$ in the SoFEA study and $25.3 \%$ (91/360) in the PALOMA3 study. PIK3CA mutations were found in the plasma of $33 \%(129 / 395)$ of patients in the PALOMA3 study [16]. They also reported the effectiveness of the target drug by having the mutations or not. In the SoFEA study, patients with ESR1 mutations had improved PFS after taking fulvestrant compared with exemestane. In the PALOMA3 study, fulvestrant plus the CDK4/6-inhibitor palbociclib improved PFS regardless of the genomic status of ESR1 or PIK3CA [15, 16].

In this retrospective study, we demonstrated the clinical significance of on-treatment hotspot ESR1 LBD mutations both in a snapshot and serially in 185 plasma samples from 86 patients in comparison with the hotspot mutation status of PIK3CA using multiplex droplet dPCR (ddPCR) assays. To our knowledge, this is the leading comparative study to identify the clinical significance of multiplex ddPCR detection of ESR 1 mutations and $P I K 3 C A$ mutations in plasma samples.

\section{RESULTS}

\section{ESR1 mutations in cfDNA baseline plasma samples}

We developed a sensitive and quantitative multiplex ddPCR assay to screen for 3 hotspot mutations in the LBD of ESR1. Figure 1A and Supplementary Figure S1 show the comparative analysis of a dilution series of each indicated synthetic ESR1 mutation oligonucleotide by ddPCR. We used serial dilutions of three hotspot ESR1 LBD mutant recombinant DNAs: ESR1 Y537S, Y 537N, and D538G, and analyzed them using a multiplex ESR 1 mutant detection probe, which could simultaneously detect ESR 1 Y537S, Y537N, and D538G, confirming that this assay was able to detect as few as three copies of the mutant allele in an abundance of wild-type DNA. There was no cross-reactivity for the detection of each ESR1 mutations.

Next, to validate the utility of the multiplex ddPCR assay, a subset of 26 women (62 blood samples), who were previously evaluated using a uniplex ESR 1 mutant detection probe [17], were analyzed using a multiplex ESR 1 mutant detection probe, and a statistically significant correlation between uniplex and multiplex ddPCR assays was found (Figure 1B, Supplementary Table S1: Y537S, $\kappa$ $=1.0, \mathrm{r}=0.91, P<0.0001 ; \mathrm{Y} 537 \mathrm{~N}, \kappa=1.0, \mathrm{r}=0.55, P<$ 0.0001 ; D538G, $\kappa=0.77, \mathrm{r}=0.71, P<0.0001)$

\section{Detection of ESR1 mutations and PIK3CA mutations in cfDNA of women with ER-positive BC}

A total of 86 patients (185 plasma samples) with breast carcinoma, who had the ECOG scale of Performance status 0 or 1 , were enrolled in this study. Participants comprised 17 women (34 plasma samples) with primary $\mathrm{BC}(\mathrm{PBC})$ and 69 women (151 plasma samples) with $\mathrm{MBC}$. The patient demographics and baseline characteristics of $\mathrm{PBC}$ and $\mathrm{MBC}$ are presented in Table 1. The median age of the patients at first blood draw was 67 years (range, 41-82) in the PBC group and 59 years (range, $32-85$ ) in the MBC group. Of the clinical stage at diagnosis $69 \mathrm{MBC}$ patients, 20 patients $(30 \%)$ were categorized as stage IV. All 17 PBC patients were treated by neoadjuvant ET. Of the MBC patients, $79.7 \%$ $(55 / 69)$ were previously treated with AIs, $60.9 \%(42 / 69)$ were previously treated with SERMs, and 56.5\% (39/69) were previously treated with both AIs and SERMs, but 
$23.2 \%(16 / 69)$ had not previously received any ET. The median duration of follow-up was 33 months (range, 13101 months) in the PBC group and 49 months (range, 11 268 months) in the MBC group. There was no recurrence during the observation period in any of the PBC patients. Concerning the multiplex assay, it should be tested using negative control to help defining the background noise [18]. Therefore, we verified the multiplex ddPCR assay using $\mathrm{PBC}$ patients whose allele frequency (AF) of ESR 1 mutations is very low [2-4]. We found 4 PIK3CA mutations in cfDNA, but we did not find any ESR 1 mutations in the PBC group (Supplementary Table S3). Figure 2 shows the percentage of ESR1 mutations and PIK3CA mutations in plasma cfDNA of BC subsets. ESR 1 mutations and $P I K 3 C A$ mutations were evaluated using multiplex mutant detection probes. ESR 1 mutations in cfDNA were detected in $30.5 \%(46 / 185)$ of all samples (Supplementary Table S2). ESR1 Y537S, Y537N, and D538G were found in $76.1 \%, 73.9 \%$, and $67.4 \%$ of samples with ESR1 mutations, respectively (Figure 2A). Among patients with any detectable ESR1 mutations, $67.4 \%(31 / 46)$ had two or more mutations $(17.4 \%(8 / 46)$ had two mutations and $50 \%(23 / 46)$ had more than three mutations). PIK3CA mutations in cfDNA were detected in $23.8 \%(44 / 185)$ of all samples (Supplementary Table S2). PIK3CA H1047L/R/Y, E545V/G/A/Q/K Q546L/R/P/E/K, $\mathrm{E} 542 \mathrm{~K} / \mathrm{V}$, and G1049R/S were found in $65.9 \%, 22.7 \%$, $20.5 \%$ and $6.8 \%$ of PIK3CA mutant samples, respectively. The majority of cfDNA samples $(88.7 \%, 39 / 44)$ had only a single-site detectable PIK3CA mutation in cfDNA and exhibited markedly less heterogeneity than ESR1 mutations (Figure 2A, Supplementary Table S2). Nine patients had co-mutation (ESR1 and PIK3CA mutations) over the all of treatment. However, there was no statistically significant correlation of copies/ $\mu \mathrm{L}$ between ESR 1 mutations and PIK3CA mutations in plasma cfDNA ( $\mathrm{r}=0.28, P=0.0001$ ) (Figure 2B, Supplementary Table $\mathrm{S} 2)$.

\section{Association of ESR1 and PIK3CA mutations with clinical features}

Table 2 shows ESR 1 and PIK3CA mutations as they relate to baseline clinical and pathological features. In the snapshot study, we analyzed the latest genetic state

A

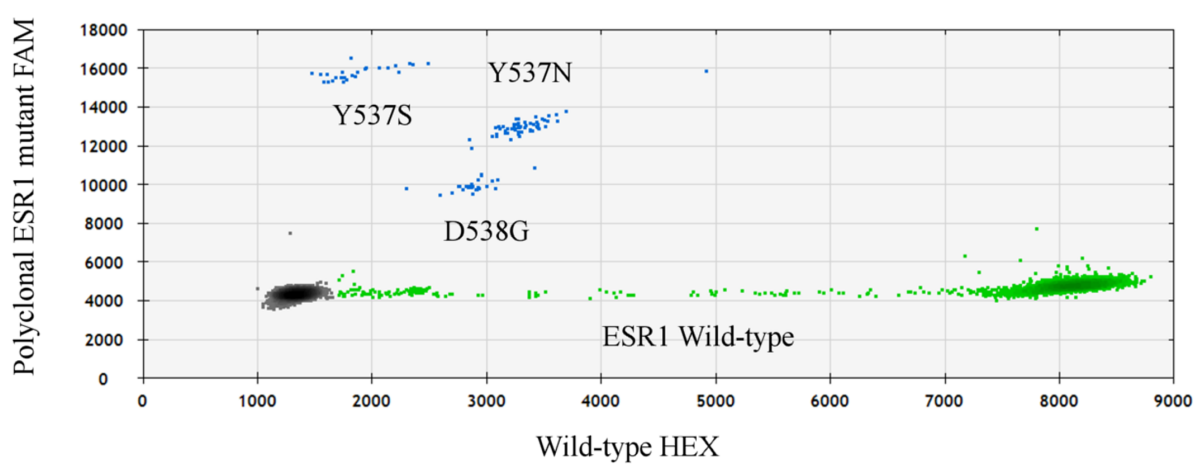

B
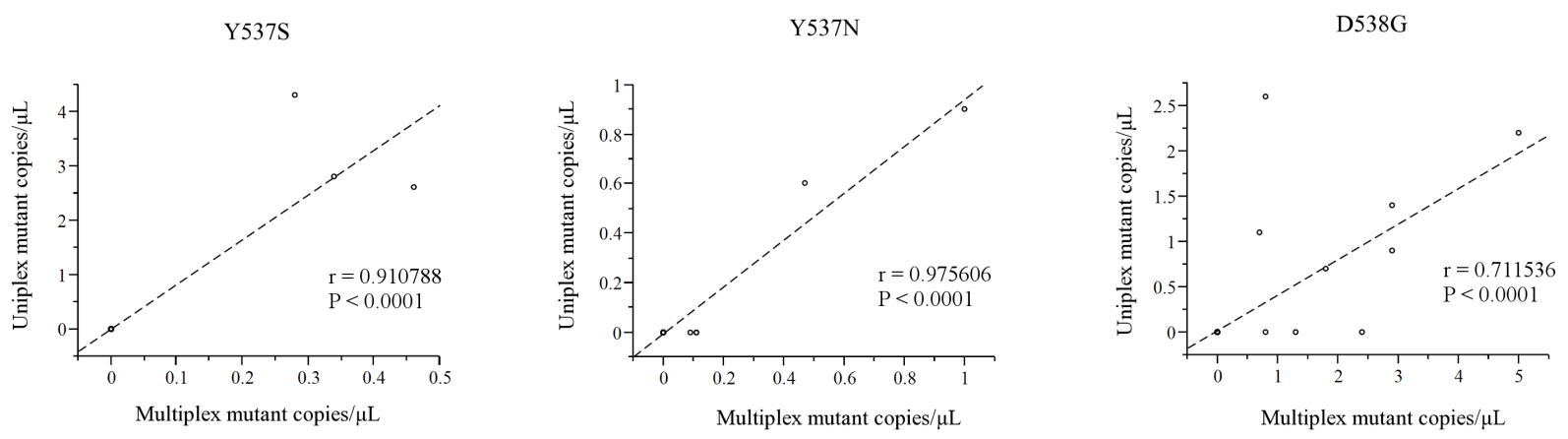

Figure 1: A. Representative ddPCR analysis of polyclonal ESR1 mutations. The presence of all three hotspot LBD ESR1 mutations (Y537S, Y537N, and D538G) was confirmed by uniplex assays (Supplementary Figure S1). In each plot, green dots represent HEX-labeled wild type DNA, blue dots represent FAM-labeled mutant DNA, and black dots are droplets with no DNA incorporated. B. Comparison of each LBD ESR1 mutation between uniplex and multiplex ddPCR assays from a validation subset of 26 women (62 blood samples). Abbreviations; ddPCR, droplet digital polymerase chain reaction; LBD, ligand binding domain; cfDNA. 
Table 1: Patient characteristics.

\begin{tabular}{|c|c|c|c|}
\hline \multirow[b]{2}{*}{ Variables } & \multicolumn{3}{|c|}{ No. of patients (\%) } \\
\hline & Total & PBC & MBC \\
\hline & $(\mathrm{N}=86)$ & $(\mathrm{N}=17)$ & $(\mathrm{N}=69)$ \\
\hline \multicolumn{4}{|l|}{ Age at biopsy } \\
\hline Median (range) & $58(31-82)$ & $67(41-82)$ & $59(32-85)$ \\
\hline \multicolumn{4}{|l|}{ Clinical Stage at diagnosis } \\
\hline $\mathrm{I}$ & $16(18.6)$ & $4(23.5)$ & $12(17.4)$ \\
\hline II & $36(41.8)$ & $11(64.7)$ & $25(36.2)$ \\
\hline III & $14(16.3)$ & $2(11.8)$ & $12(17.4)$ \\
\hline IV & $20(23.3)$ & 0 & $20(30)$ \\
\hline \multicolumn{4}{|l|}{ Histological type } \\
\hline Invasive ductal & $79(91.9)$ & $13(76.5)$ & $66(95.5)$ \\
\hline Invasive lobular & $2(2.3)$ & $1(5.9)$ & $1(1.5)$ \\
\hline Mucinous & $4(4.7)$ & $3(17.6)$ & $1(1.5)$ \\
\hline Neuroendocrine therapy & $1(1.1)$ & 0 & $1(1.5)$ \\
\hline \multicolumn{4}{|l|}{ Histological grade } \\
\hline 1 & $27(31.4)$ & $5(29.4)$ & $22(31.9)$ \\
\hline 2 & $37(43.0)$ & $11(64.7)$ & $26(37.7)$ \\
\hline 3 & $16(18.6)$ & 0 & $16(23.2)$ \\
\hline Lobular & $2(2.3)$ & $1(5.9)$ & $1(1.4)$ \\
\hline Unknown & $4(4.7)$ & 0 & $4(5.8)$ \\
\hline Percentage of ER $\alpha$ median $(25 \%, 75 \%)$ & 86 & $90(90-95)$ & $90(65-95)$ \\
\hline Percentage of PgR median $(25 \%, 75 \%)$ & 86 & $50(7.5-80)$ & $30(0-70)$ \\
\hline \multicolumn{4}{|l|}{ HER2 } \\
\hline Negative & $76(88.4)$ & $16(94.1)$ & $60(87)$ \\
\hline Positive & $10(11.6)$ & $1(5.9)$ & $9(13)$ \\
\hline \multicolumn{4}{|l|}{ Visceral involvement } \\
\hline No & $25(29.1)$ & $17(100)$ & $18(26.1)$ \\
\hline Yes & $51(70.9)$ & 0 & $51(73.9)$ \\
\hline \multicolumn{4}{|l|}{ Bone involvement } \\
\hline No & $48(55.8)$ & $17(100)$ & $31(44.9)$ \\
\hline Yes & $38(44.2)$ & 0 & $38(55.1)$ \\
\hline \multicolumn{4}{|l|}{ Number of metastatic lesions } \\
\hline 0 & $17(19.8)$ & $17(100)$ & 0 \\
\hline 1 & $8(9.3)$ & 0 & $8(11.6)$ \\
\hline 2 & $25(29.1)$ & 0 & $25(36.2)$ \\
\hline $3 \leq$ & $36(41.8)$ & 0 & $36(52.2)$ \\
\hline \multicolumn{4}{|l|}{ Prior endocrine therapy } \\
\hline SERM & $42(48.8)$ & 0 & $42(60.9)$ \\
\hline $\mathrm{AI}$ & $72(83.7)$ & $17(100)$ & $55(79.7)$ \\
\hline Both AI and SERM & $39(45.3)$ & 0 & $39(56.5)$ \\
\hline \multicolumn{4}{|l|}{ Number of prior endocrine regimens } \\
\hline 0 & $16(18.6)$ & 0 & $16(23.2)$ \\
\hline 1 & $29(33.7)$ & $17(100)$ & $12(17.4)$ \\
\hline 2 & $5(5.8)$ & 0 & $5(7.3)$ \\
\hline $3 \leq$ & $36(41.9)$ & 0 & $36(52.1)$ \\
\hline \multicolumn{4}{|l|}{ Number of prior courses of chemotherapy } \\
\hline 0 & $52(60.5)$ & $17(100)$ & $35(50.7)$ \\
\hline 1 & $16(18.6)$ & 0 & $16(23.2)$ \\
\hline 2 & $3(3.5)$ & 0 & $3(4.4)$ \\
\hline $3 \leq$ & $15(17.4)$ & 0 & $15(21.7)$ \\
\hline
\end{tabular}

Abbreviations: $\mathrm{PBC}$, primary breast cancer; $\mathrm{MBC}$, metastatic breast cancer; ER $\alpha$, estrogen receptor alpha; PgR, progesterone receptor; HER2, human epidermal growth factor receptor 2; SERM, selective estrogen receptor modulator; AI, aromatase inhibitor. 
in cfDNA in each BC patient. Plasma ESR1 mutations were found in $28.9 \%(20 / 69)$ of MBC patients while plasma PIK3CA mutations were found in $24.6 \%(17 / 69)$. The presence of plasma ESR 1 mutations was associated with several clinicopathological parameters. Ninety-five percent of patients with ESR1 mutations had visceral disease, whereas only $65.4 \%$ of ESR 1 wild-type (WT) patients had visceral disease $(P=0.011)$. In addition, all patients with ESR 1 mutations had resistance to prior AI therapy compared with $71.4 \%$ of ESR1 WT patients $(P=0.0074) ; 85 \%$ of patients with ESR 1 mutations had resistance to prior SERM therapy compared with $51 \%$ of ESR 1 WT patients $(P=0.0087)$, and $80 \%$ of patients with ESR 1 mutations had been treated with a prior endocrine regimen three or more times compared with $40.3 \%$ of ESR1 WT patients. Other demographic and clinical parameters were generally balanced between patients with ESR1 mutations and ESR1 WT patients, including age, histological grade, the expression of ER and progesterone receptor (PgR), human epidermal growth factor receptor 2 (HER2) positivity, bone involvement, the number of metastatic lesions, the number of subsequent endocrine regimens, and the number of prior and subsequent chemotherapies and total duration of chemotherapies. In similar analyses, PIK3CA mutations in cfDNA generally were not associated with particular demographic and clinical features, with the exception of visceral involvement $(P=0.029)$. In the PBC group, the presence of PIK3CA mutations in cfDNA was not associated with particular clinicopathological features (Supplementary Table S3).

\section{Association of ESR1 and PIK3CA mutations with clinical outcome}

We retrospectively analyzed whether ESR 1 and $P I K 3 C A$ mutations detected in cfDNA were associated with differential benefit in relation to the duration of ET effectiveness (Figure 3, Supplementary Figure S2). In that analysis, discontinuation of ETs caused by local recurrences, distant metastases, and disease progression at any site following the blood draw were considered as an event. These were tested by Kaplan-Meier analysis and verified by the log-rank test. Patients with detectable plasma ESR1 mutations $(P<0.0001)$ and PIK3CA mutations $(P=0.0034)$ showed statistically significant shorter duration of ET effectiveness (Figure 3A, 3B). The Cox hazards model analysis of duration of ET effectiveness is shown in Table 3 . The presence of ESR1 mutations in cfDNA was a significant prognostic parameter in univariate analysis (hazard ratio (HR): 3.2, $95 \%$ confidence interval $(\mathrm{CI}): 1.76-5.71, P=0.0002)$ and
A

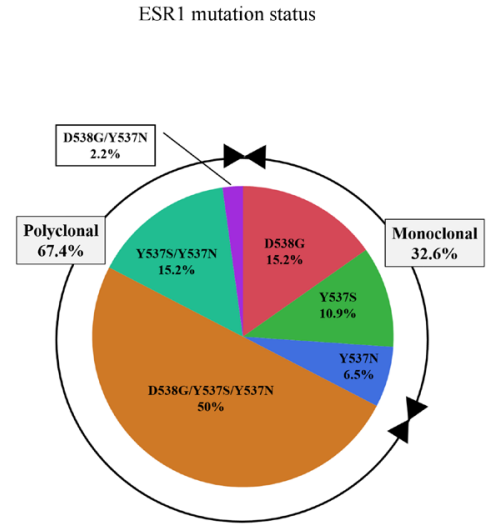

B

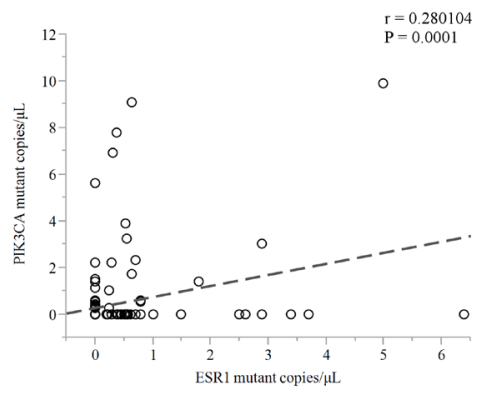

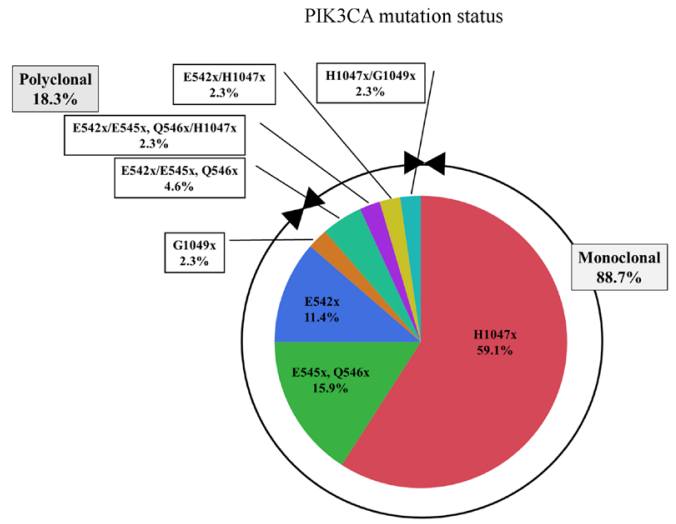

Figure 2: A. Chart showing the percentage of ESR1 mutations and PIK3CA mutations in plasma cfDNA in this cohort. B. Comparison of the number of copies/ $\mu \mathrm{L}$ between total ESR1 mutations and total PIK3CA mutations in plasma from a subset of 86 women (185 blood samples). Abbreviations; cfDNA, cell-free DNA; H1047x, H1047L/R/Y; E545x Q546x, E545V/G/A/Q/K Q546L/R/P/E/K; E542x, E542K/V; G1049x, G1049R/S. 
Table 2: Patients and clinicopathological characteristics associated with $E S R 1$ and $P I K 3 C A$ mutations in cfDNA of 69 MBC patients.

\begin{tabular}{|c|c|c|c|c|c|c|}
\hline \multirow{3}{*}{ Variables } & \multicolumn{6}{|c|}{ No. of patients $(\%)$} \\
\hline & \multicolumn{2}{|l|}{ ESR1 } & \multirow{2}{*}{$P$-value } & \multicolumn{2}{|l|}{ PIK3CA } & \multirow{2}{*}{$P$-value } \\
\hline & wild type & mutation & & wild type & mutation & \\
\hline & $(N=49)$ & $(N=\mathbf{2 0})$ & & $(N=52)$ & $(N=17)$ & \\
\hline \multicolumn{7}{|l|}{ Age at biopsy } \\
\hline Median (range) & $59(50.5-65)$ & $60(51.3-70.5)$ & 0.30 & $58.5(51-66)$ & $61(48.5-65.5)$ & 0.92 \\
\hline \multicolumn{7}{|l|}{ Histological grade } \\
\hline 1 & $18(38.3)$ & $4(23.5)$ & 0.55 & $14(26.9)$ & $8(47.1)$ & 0.24 \\
\hline 2 & $18(38.3)$ & $8(47.1)$ & & $22(42.3)$ & $4(23.5)$ & \\
\hline 3 & $11(23.4)$ & $5(29.4)$ & & $12(23.1)$ & $4(23.5)$ & \\
\hline Median percentage of $\mathrm{ER} \alpha(25 \%, 75 \%)$ & $90(70-95)$ & $90(52.5-100)$ & 0.85 & $90(60-95)$ & $90(80-95)$ & 0.54 \\
\hline Median percentage of $\operatorname{PgR}(25 \%, 75 \%)$ & $40(0-70)$ & $7.5(0-70)$ & 0.41 & $40(0-70)$ & $5(0-75)$ & 0.63 \\
\hline HER2-positive & $6(12.2)$ & $4(20)$ & 0.41 & $8(15.3)$ & $2(11.8)$ & 0.71 \\
\hline Visceral involvement & $32(65.3)$ & $19(95)$ & $0.011 \mathrm{a}$ & $35(67.3)$ & $16(94.1)$ & $0.029 \mathrm{a}$ \\
\hline Bone involvement & $26(53.1)$ & $12(60)$ & 0.6 & $29(55.8)$ & $9(52.9)$ & 0.84 \\
\hline \multicolumn{7}{|l|}{ Number of metastatic lesions } \\
\hline 1 & $8(16.3)$ & 0 & 0.12 & $7(13.5)$ & $1(5.9)$ & 0.93 \\
\hline 2 & $18(36.7)$ & $7(35)$ & & $19(36.5)$ & $6(36.2)$ & \\
\hline $3 \leq$ & $23(47)$ & $13(65)$ & & $26(50)$ & $10(58.8)$ & \\
\hline \multicolumn{7}{|l|}{ Prior endocrine therapy } \\
\hline SERM & $25(51)$ & $17(85)$ & $0.0087 \mathrm{a}$ & $29(55.8)$ & $13(76.5)$ & 0.12 \\
\hline $\mathrm{AI}$ & $35(71.4)$ & $20(100)$ & $0.0074 \mathrm{a}$ & $40(76.9)$ & $15(88.2)$ & 0.31 \\
\hline Both AI and SERM & $22(44.9)$ & $17(85)$ & $0.0014 \mathrm{a}$ & $27(51.9)$ & $12(70.6)$ & 0.17 \\
\hline \multicolumn{7}{|l|}{ Number of prior endocrine regimens } \\
\hline 0 & $16(32.7)$ & 0 & $0.041 \mathrm{a}$ & $15(28.8)$ & $1(5.9)$ & 0.052 \\
\hline 1,2 & $13(26.5)$ & $4(20)$ & & $14(26.9)$ & $3(17.6)$ & \\
\hline $3 \leq$ & $20(40.8)$ & $16(80)$ & & $23(44.2)$ & $13(76.5)$ & \\
\hline \multicolumn{7}{|l|}{ Number of subsequent endocrine regimen } \\
\hline 0 & $4(8.2)$ & $5(25)$ & 0.16 & $5(28.8)$ & $4(23.5)$ & 0.11 \\
\hline 1,2 & $22(44.9)$ & $8(40)$ & & $21(26.9)$ & $9(17.6)$ & \\
\hline $3 \leq$ & $23(46.9)$ & $7(35)$ & & $26(44.2)$ & $4(76.5)$ & \\
\hline \multicolumn{7}{|l|}{ Number of prior courses of chemotherapy } \\
\hline 0 & $25(51)$ & $10(50)$ & 0.95 & $30(57.7)$ & $5(29.4)$ & 0.13 \\
\hline 1,2 & $13(26.5)$ & $6(30)$ & & $12(23.1)$ & $7(41.2)$ & \\
\hline $3 \leq$ & $11(22.4)$ & $4(20)$ & & $10(19.2)$ & $5(29.4)$ & \\
\hline \multicolumn{7}{|c|}{ Number of subsequent courses of chemotherapy } \\
\hline 0 & $18(36.7)$ & $7(35)$ & 0.40 & $19(36.5)$ & $6(35.3)$ & 0.96 \\
\hline 1,2 & $21(42.9)$ & $6(30)$ & & $21(40.4)$ & $6(35.3)$ & \\
\hline $3 \leq$ & $10(20.4)$ & $7(35)$ & & $12(23.1)$ & $5(29.4)$ & \\
\hline \multicolumn{7}{|l|}{ Duration of total chemotherapy (months) } \\
\hline Median (range) & $13(0-118)$ & $12.5(0-38)$ & 0.90 & $13(0-60)$ & $14(0-118)$ & 0.69 \\
\hline
\end{tabular}

Abbreviations: ER $\alpha$, estrogen receptor alpha; PgR, progesterone receptor; HER2, human epidermal growth factor receptor 2; SERM, selective estrogen receptor modulator; AI, aromatase inhibitor.

a Factor showing statistical significance $(P<0.05)$.

in multivariate analysis (HR: $2.04,95 \% \mathrm{CI}: 1.08-3.83, P=$ 0.029). The presence of $P I K 3 C A$ mutations in cfDNA was a significant prognostic parameter in univariate analysis (HR: 2.25, 95\% CI: 1.24-3.94, $P=0.0091$ ), but showed only a marginal relationship in multivariate analysis (HR: $1.78,95 \%$ CI: 0.96-3.18, $P=0.066$ ). Other clinical parameters were not found to be statistically significant in univariate analysis, with the exception of prior SERM (HR: $2.30,95 \% \mathrm{CI}: 1.39-3.87, P=0.0011$ ), prior AI (HR: 2.02, 95\% CI: $1.14-3.81, P=0.015)$, and prior both AIs and SERMs (HR: 2.89 , 95\% CI: 1.73-4.92, $P<0.0001$ ), nor in multivariate analysis. We also examined whether 
Table 3: Univariate and multivariate analysis of factors associated with discontinuity in endocrine therapy in women with breast cancer.

\begin{tabular}{|c|c|c|c|c|c|c|c|c|}
\hline \multirow[t]{2}{*}{ Variables } & & & \multicolumn{3}{|c|}{ Univariate analysis } & \multicolumn{3}{|c|}{ Multivariate analysis } \\
\hline & & Value & HR & $95 \% \mathrm{CI}$ & $P$ value & HR & $95 \%$ CI & $P$ value \\
\hline Age at biopsy & $($ ref $=\leq 50)$ & $>50$ & 1.26 & $0.72-2.34$ & 0.43 & & & \\
\hline Histological grade & $(\mathrm{ref}=1,2)$ & 3 & 0.96 & $0.52-1.69$ & 0.91 & & & \\
\hline ER (IHC) & $($ ref $=$ median $)$ & $\geq 90 \%$ & 1.28 & $0.77-2.10$ & 0.34 & & & \\
\hline PgR (IHC) & $($ ref $=$ median $)$ & $\geq 30 \%$ & 0.99 & $0.61-1.60$ & 0.97 & & & \\
\hline Visceral involvement & $(\mathrm{ref}=\mathrm{No})$ & Yes & 1.04 & $0.61-1.84$ & 0.90 & & & \\
\hline Bone involvement & $(\mathrm{ref}=\mathrm{No})$ & Yes & 1.22 & $0.76-1.99$ & 0.41 & & & \\
\hline Number of metastatic lesions & $(\mathrm{ref}=1,2)$ & $\geq 3$ & 1.20 & $0.74-1.94$ & 0.46 & & & \\
\hline \multicolumn{9}{|l|}{ Prior endocrine therapy } \\
\hline SERM & $(\mathrm{ref}=\mathrm{No})$ & Yes & 2.30 & $1.39-3.87$ & $0.0011^{\mathrm{a}}$ & 0.70 & $0.16-2.3$ & 0.59 \\
\hline$\overline{\mathrm{AI}}$ & $(\mathrm{ref}=\mathrm{No})$ & Yes & 2.02 & $1.14-3.81$ & $0.015^{\mathrm{a}}$ & 1.0 & $0.46-2.3$ & 0.99 \\
\hline Both AI and SERM & $(\mathrm{ref}=\mathrm{No})$ & Yes & 2.89 & $1.73-4.92$ & $<0.0001^{\mathrm{a}}$ & 2.96 & $0.75-15.1$ & 0.13 \\
\hline ESR1 genomic state & $($ ref $=W T)$ & Mut & 3.2 & $1.76-5.71$ & $0.0002^{\mathrm{a}}$ & 2.04 & $1.08-3.83$ & $0.029^{\mathrm{a}}$ \\
\hline ESR1 MAF (\%) & $($ ref $=<$ Median $)$ & Median > & 0.73 & $0.30-1.82$ & 0.51 & & & \\
\hline$P I K 3 C A$ genomic state & $(\mathrm{ref}=\mathrm{WT})$ & Mut & 2.25 & $1.24-3.94$ & $0.0091^{\mathrm{a}}$ & 1.78 & $0.96-3.18$ & 0.066 \\
\hline PIK3CA МАF (\%) & (ref $=<$ Median) & Median > & 0.76 & $0.29-2.04$ & $0.58^{\mathrm{a}}$ & & & \\
\hline
\end{tabular}

(Cox proportional hazards model)

Abbreviations: HR, hazard ratio; 95\%CI, 95\% confidence interval; ER, estrogen receptor; IHC, immunohistochemistry; PgR, progesterone receptor; SERM, selective estrogen receptor modulator; AI, aromatase Inhibitor; MAF, mutant allele frequency; Mut, mutation.

${ }^{a}$ Factor showing statistical significance.



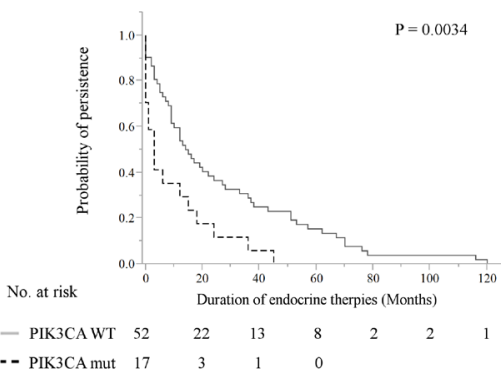

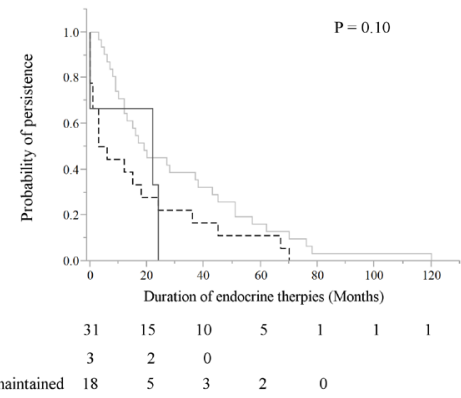

Figure 3: Kaplan-Meier plots of the association of ESRI mutations and PIK3CA mutations in cfDNA with duration of ET effectiveness in this cohort. These were verified by the log-rank test. A, B. Patients with detectable plasma ESR1 mutations $(P$ $<0.0001)(\mathrm{A})$ and $P I K 3 C A$ mutations $(P=0.0034)(B)$ showed significantly shorter duration of ET effectiveness in $69 \mathrm{MBC}$ patients. C., D. Tracking analysis of cfDNA ESR1 mutations and PIK3CA mutations in 52 breast cancer patients with longitudinal samples. Patients were grouped into those who did not have any ESR1 or PIK3CA mutations over the course of treatment, those in whom ESR1 or PIK3CA mutations were maintained or acquired, and those in whom ESRI or PIK3CA mutations disappeared in cfDNA. C. Patients in the loss of cfDNA ESR1 mutations group had a longer duration of ET effectiveness than patients with acquired or maintained numbers of cfDNA ESR1 mutations, but had a shorter duration of ET effectiveness than patients without mutations over the course of treatment $(P<0.0001)$. D. There was no statistically significant differences in these three groups; no PIK3CA mutations during treatment group, the loss of cfDNA PIK3CA mutations group, and the acquired or maintained numbers of cfDNA PIK3CA mutations group. Abbreviations: ET, endocrine therapy; cfDNA, cell-free DNA; MBC, metastatic breast cancer. 
patients with a higher AF of ESR 1 mutations or PIK3CA mutations in cfDNA showed differential outcomes in duration of ET effectiveness. The box-plots for the AF of ESR 1 mutations and PIK3CA mutations are shown in Supplementary Figure S2. The median AF of ESR1 mutations was $8.66 \%$ (range, $0.35-78.5$ ) and the median AF of PIK3CA mutations was $10.3 \%$ (range, 2.57-35), respectively. The dichotomized ESR1 and PIK3CA AF (cutoff each AF $>$ median) did not show a clear difference in duration of ET effectiveness.
A. AI
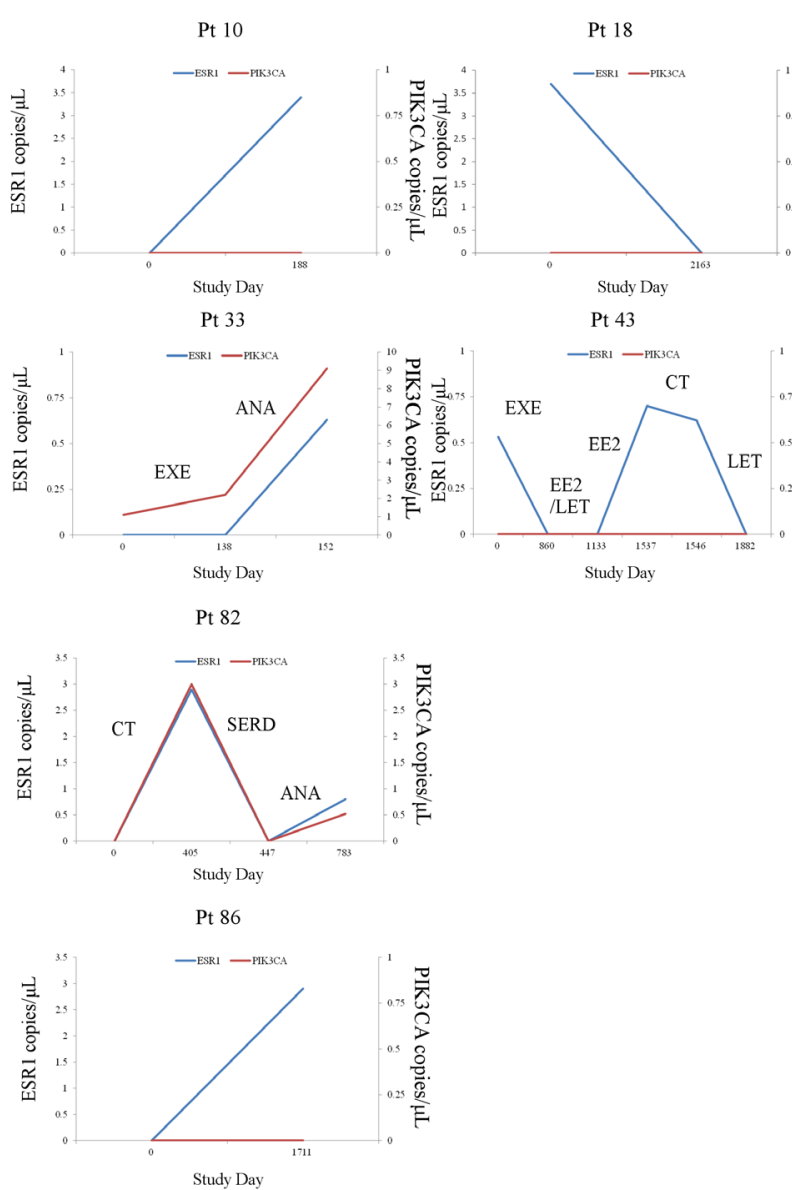

C. SERM
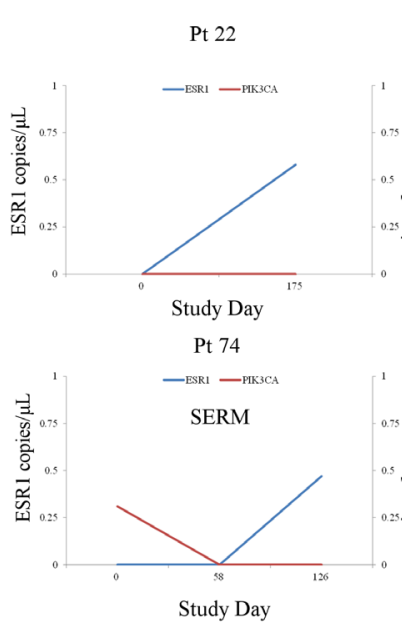

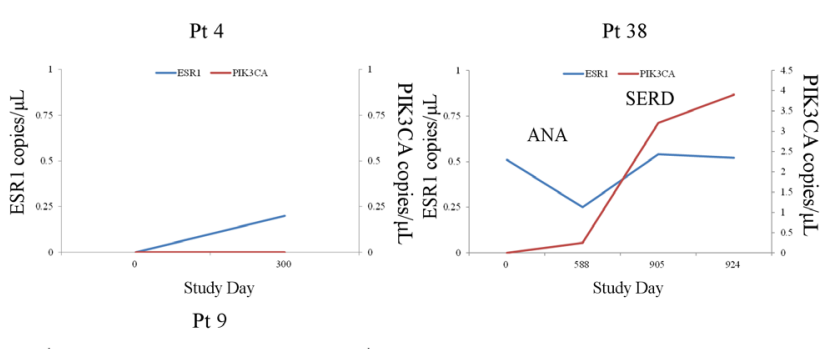

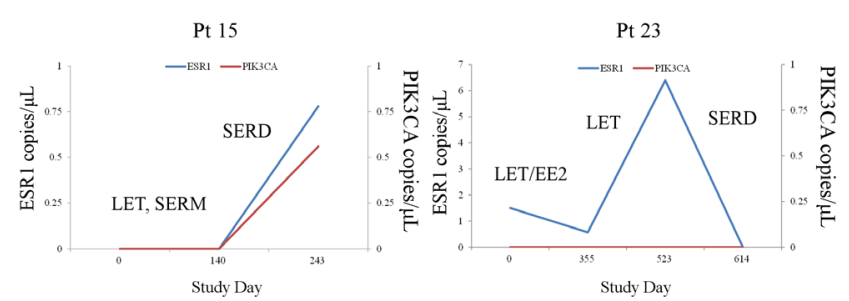

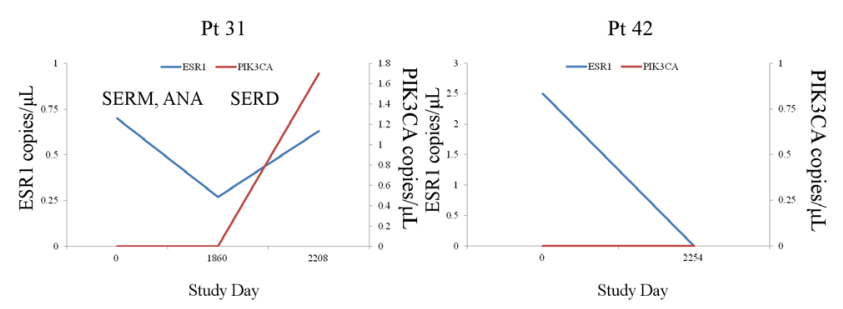

D. EE2
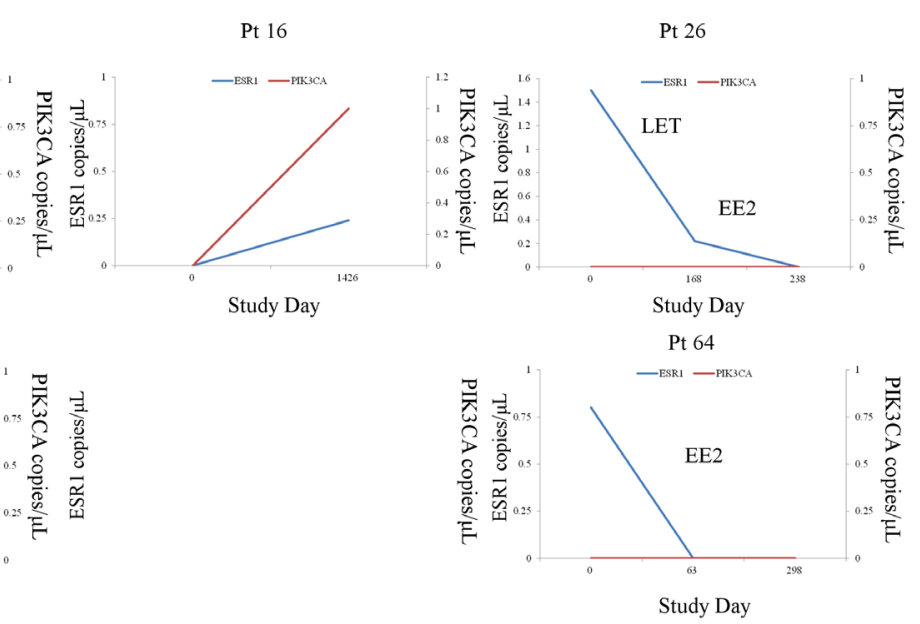

Figure 4: A.-D. Intrapatient changes of the numbers of copies/ $\mu \mathrm{L}$ of $E S R 1$ and PIK3CA mutations under treatment with each representative therapeutic drug in 21 patients with longitudinal data. A. Patients treated with AIs, B. Patients treated with SERDs, C. Patients treated with SERMs, D. Patients treated with EE2. Abbreviations; AI, aromatase inhibitor; SERD, selective estrogen receptor downregulator; SERM, selective estrogen receptor modulator; EE2, ethinyl estradiol. 
Table 4: Comparison between changes of ESR1 and PIK3CA mutations and each therapeutic drug in $52 \mathrm{MBC}$ patients with longitudinal data.

\begin{tabular}{|c|c|c|c|c|c|c|c|c|c|}
\hline & \multirow{4}{*}{ Genomic state } & \multicolumn{8}{|c|}{ No. of patients $(\%)$} \\
\hline & & \multicolumn{8}{|c|}{ Representative treatment between blood draws } \\
\hline & & Total & No & AI & SERM & SERD & EE2 & MPA & Chemotherapy \\
\hline & & $(N=52)$ & $(N=7)$ & $(N=15)$ & $(N=5)$ & $(N=8)$ & $(N=5)$ & $(N=2)$ & $(N=10)$ \\
\hline \multirow{3}{*}{ ESR1 } & WT & $28(53.8)$ & $7(100)$ & $9(60)$ & $1(20)$ & $1(12.5)$ & $2(40)$ & $1(50)$ & $7(70)$ \\
\hline & $\begin{array}{l}\text { Acquires or maintenance of } \\
\text { mutation numbers }\end{array}$ & $15(28.8)$ & 0 & $4(26.7)$ & $2(40)$ & $5(62.5)$ & $1(20)$ & $1(50)$ & $2(20)$ \\
\hline & \begin{tabular}{|l|l|}
$\begin{array}{l}\text { Disappears in mutation } \\
\text { numbers }\end{array}$ & . \\
\end{tabular} & $9(17.3)$ & 0 & $2(13.3)$ & $2(40)$ & $2(25)$ & $2(40)$ & 0 & $1(10)$ \\
\hline \multirow{3}{*}{ PIK3CA } & WT & $31(59.6)$ & $7(100)$ & $10(75)$ & $3(60)$ & $5(62.5)$ & $2(40)$ & $1(50)$ & $3(30)$ \\
\hline & $\begin{array}{l}\text { Acquires or maintenance of } \\
\text { mutation numbers }\end{array}$ & $18(34.6)$ & 0 & $5(25)$ & $1(20)$ & $3(37.5)$ & $3(60)$ & $1(50)$ & $5(50)$ \\
\hline & \begin{tabular}{|l|}
$\begin{array}{l}\text { Disappears in mutation } \\
\text { numbers }\end{array}$ \\
\end{tabular} & $3(5.8)$ & 0 & 0 & $1(20)$ & 0 & 0 & 0 & $2(20)$ \\
\hline
\end{tabular}

Abbreviations: WT, wild-type; AI, aromatase inhibitor; SERM, selective estrogen receptor modulator; SERD, .selective estrogen receptor downregulator; EE2, Ethinyl estradiol; MPA, medroxyprogesterone acetate.

\section{Tracking cfDNA ESR1 mutations in $52 \mathrm{MBC}$ patients with longitudinal samples}

Longitudinal plasma samples, collected at more than two time-points of the clinical course, from 52 patients were used to look at changes in the presence of ESRI and PIK3CA mutations during treatment (three points from a total of 12 patients $(23.1 \%)$, four points from a total of $5(9.6 \%)$, five points from one $(1.9 \%)$, and six points from one (1.9\%) out of $52 \mathrm{MBC}$ patients). The actual changes in the number of copies/ $\mu \mathrm{L}$ in ESR1 mutations and $P I K 3 C A$ mutations in cfDNA during treatment in $52 \mathrm{MBC}$ patients are shown in Supplementary Figure S3 Fourteen patients had ESR1 mutations and 9 patients had PIK3CA mutations in the first blood draw. Over the course of treatment, 10 patients acquired, but 9 patients lost ESR1 mutations. Meanwhile, 12 patients acquired, but 3 patients lost PIK3CA mutations. Thus, there are more ESR 1 and PIK3CA mutated patients in the serial analysis than in the snap shot analysis (Supplementary Table S4). Patients were grouped into those who had no ESR1 or PIK3CA mutations over the course of treatment $(N=28)$, those in whom ESRI or PIK3CA mutations were acquired or maintained $(N=15)$, and those in whom ESRI or PIK3CA mutations were disappeared after treatment in cfDNA $(N=9)$, and groups were compared by the patient response end-points of duration of ET effectiveness (Figure 3C, 3D). Patients in the loss of cfDNA ESR1 mutations group had a longer duration of ET effectiveness than patients in the acquired or maintained numbers of cfDNA ESR1 mutations group, but had a shorter duration of ET effectiveness than patients without mutations over the course of treatment $(P<0.0001)$. On the other hand, there was no statistically significant differences in these three groups; no PIK3CA mutations during treatment group $(N=31)$, the loss of cfDNA PIK3CA mutations group $(N=3)$, and the acquired or maintained numbers of cfDNA PIK3CA mutations group $(N=18)(P=0.10)$. We did not detect any trend towards particular ESR1 and PIK3CA mutation hotspots over the course of treatment, but indicated that polyclonal mutations appeared or disappeared more frequently in ESR1 than in PIK3CA (Supplementary Table S4; appeared polyclonal mutations, $40 \%$ for ESR1 vs $5.6 \%$ for PIK3CA: disappeared polyclonal mutations, $66.7 \%$ for $E S R 1$ vs $0 \%$ for $P I K 3 C A$ ). Intrapatient changes of the number of copies $/ \mu \mathrm{L}$ of ESRI and PIK3CA mutations in each representative therapeutic drug group in 21 patients with longitudinal data are shown in Figure 4. Furthers, we listed the treatment just before the latest blood draw as "representative treatment between blood draw" in Table 4. All plasma samples were taken at the time of disease progression, so that it provided an evaluation of ESR1 and PIK3CA mutation status when each ET failed. In patients treated with AIs, 26.7\% (4/15) had acquired ESR1 mutations, which was more frequent compared to the $13.3 \%(2 / 15)$ of patients lost ESR1 mutations. The two patients acquiring ESR1 mutations (Pt 33 and Pt 82) developed PIK3CA mutations over the course of treatment. In patients treated with selective ER down regulators (SERDs), 62.5\% (5/8) showed acquired or maintained numbers of ESR1 mutations, but $25 \%$ $(2 / 8)$ had decreases in the number of ESRI mutations. Of the SERD-treated patients acquiring or maintaining ESR1 mutations, $60 \%(3 / 5)$ showed increases in PIK3CA mutations. In patients treated with SERMs, 50\% (2/4) had acquired ESR1 mutations. In patients treated with ethinyl estradiol (EE2), 40\% (2/5) of EE2-treated patients showed decreases in ESR1 mutations. Interestingly, among ETs, decreases in PIK3CA mutations were detected in one 
SERM-treated patient.

\section{DISCUSSION}

dPCR is a highly sensitive technique for detecting rare mutations, but analysis is on the basis of a single mutation per assay. For screening multiple mutations in a limited amount of sample, an assay to detect multiple mutations in parallel has been demonstrated with dPCR $[15,19,20]$. Here, we used multiplex ddPCR to study a cohort of patients treated with multiple lines of hormonal therapy to determine whether on-treatment ESR1 and PIK3CA mutations can be detected noninvasively and to examine the potential clinical importance of these mutations both in a snapshot and serially.

We verified that up to three mutations in ESRI and up to 12 and five mutations in PIK3CA could be multiplexed into a single assay, which could detect the three most common mutations in ESR1 (Y537S, Y537N, and $\mathrm{D} 538 \mathrm{G}$ ) and 17 mutations in the 5 most common mutation sites of $P I K 3 C A(\mathrm{E} 542 \mathrm{~K} / \mathrm{V}, \mathrm{E} 545 \mathrm{~V} / \mathrm{G} / \mathrm{A} / \mathrm{Q} / \mathrm{K}$, Q546L/R/P/E/K, H1047L/R/Y, G1049R/S) (Figure 1, Supplementary Figure S4). Toy and colleagues revealed the spectrum of ESR1 mutations from more than 900 patients and their potential differential impact (e.g. tumors driven by Y537S, but not D538G, E380Q, or S463P, were less effectively inhibited by fulvestrant in comparison with more potent and bioavailable antagonists, including AZD9496 [21]. Therefore, we examined the less frequent ESR1 mutations, E380Q $\left(\mathrm{LBx}^{\circledR}\right.$ Probe ESR1 E380Q (A081), Riken Genesis, Tokyo, Japan) and Y537C (probe shown in [22]), but we could not detect them in our cohort. In validation assay, some of the mutations are found in the multiplex assay, but not using the uniplex assay (Y537N: 5 detection by multiplex, only 2 by uniplex) (Supplementary Table S1). Because the cut-off level of the presence of the mutation was 3 positive droplets in both multiplex and uniplex assay, this study had the possibility that uniplex assay could not detect a mutation due to less than the cut-off level, which could be detected as one of target mutations using multiplex probe.

The subjects of this retrospective study were a total of 185 plasma samples from 86 ER-positive patients, of which 151 plasma samples were from $69 \mathrm{MBC}$ patients and 34 plasma samples were from 17 advanced PBC patients. Plasma ESR 1 mutations were found in $28.9 \%$ (20/69) of MBC patients while plasma PIK3CA mutations were found in $24.6 \%$ (17/69) of MBC patients, with a distribution of mutations that was highly similar to previously published data [2-4, 19]. Interestingly, only 9 patients had both ESR 1 and PIK3CA mutations over the all of treatment. These results were compatible with the recent report that $P I K 3 C A$ was mutated in $37 \%(53 / 143)$ and ESR 1 was mutated in 14\% (20/143) of the ER-positive /HER2-negative MBC, in which only 9 had both mutations [23].
All patients with ESR1 mutations had resistance to prior AI $(P=0.0074)$, and the majority of patients with ESR1 mutations had resistance to prior SERM therapy $(P=0.0087)$, prior both $\mathrm{AI}$ and SERM therapy $(P=$ $0.0014)$, and had received three or more prior endocrine regimens $(P=0.041)$ (Table 2$)$. Detailed information of three or more prior endocrine regimens was shown in Supplementary Table S5. On the other hand, PIK3CA mutations in cfDNA were not associated with previous endocrine exposure. Among all samples, 67.4\% had polyclonal mutations in ESR1, which exhibited markedly more heterogeneity than PIK3CA mutations, $11.3 \%$ of which were polyclonal (Figure 2A). These findings are compatible with the report that polyclonal ESR 1 mutations were present in $19.2-49.1 \%$ of ESR 1 mutant patients [13, $15,19,24]$, but PIK3CA mutations were often monoclonal $[15,25]$.

In this study, we focused on "duration" of ET effectiveness. Because endocrine treatments can be administered repeatedly and consistently in ER-positive MBC patients without life-threating visceral metastases [26], the efficacy of endocrine treatments may contribute to the "duration" of ET effectiveness even if the endocrine treatments during the period varied. Furthers, this analysis had the problem that the time points and the number of samples analyzed were different among the patients. However, we overwhelmed it by comparing ESR 1 mutations with PIK3CA mutations because the clinical role of PIK3CA mutations becomes clear as follow; PIK3CA mutations have the highest frequency in primary and metastatic breast tumors [12] and they are not statistically significant prognostic marker or predictor of ET effectiveness $[14,16]$. Patients with detectable plasma ESR 1 mutations $(P<0.0001)$ and PIK3CA mutations $(P$ $=0.0034)$ showed significantly shorter duration of ET effectiveness by log-rank test (Figure 3, Supplementary Figure S2). In the Cox hazards model, the presence of ESR1 mutations in cfDNA was a significant prognostic parameter in both univariate analysis (HR: 3.2, 95\% CI: $1.76-5.71, P=0.0002)$ and in multivariate analysis (HR: 2.04, 95\% CI: 1.08-3.83, $P=0.029$ ). However, the presence of PIK3CA mutations in cfDNA was a significant prognostic parameter in univariate analysis only (Table 3 ).

Potential interest of monitoring ESR 1 mutations in the metastatic setting has been increasing. Recently, Clatot and colleagues reported that cfDNA ESR1 mutations are independent risk factors for poor outcome after AI failure, and they are frequently detectable before clinical progression [27]. On the other hand, Spoerkle and colleagues did not show clinical utility of ESR1 mutations as a monitoring tool [24]. In our tracking cfDNA ESR1 mutations and PIK3CA mutations study, patients in whom the number of cfDNA ESR1 mutations were lost had a longer duration of ET effectiveness than patients in whom the numbers of cfDNA ESR1 mutations were acquired or maintained, but had a shorter duration of ET effectiveness 
than patients without mutations over the course of treatment $(P<0.0001)$. On the other hand, there was no statistically significant differences in these three groups; no PIK3CA mutations during treatment group $(N=31)$, the loss of cfDNA PIK3CA mutations group $(N=3)$, and the acquired or maintained numbers of cfDNA $P I K 3 C A$ mutations group $(N=18)(P=0.10)$. These differences regarding the prevalence of ESR 1 and $P I K 3 C A$ mutations may be caused by polyclonal breast tumor evolution under the selective pressure of ET [24]. ESR1 mutations occur late in endocrine treatment and in a subclonal manner, so that these mutations are generally detected in metastatic lesions [2-4, 22]. In contrast, most PIK3CA mutations occur early in the process of tumor development and its status does not change in the majority of patients who develop recurrent or progressive breast cancer [28] (Figure 4).

The present study has limitations. This was a retrospective, single-institute study, and was prone to selection bias. The studied population was heterogeneously treated and all plasma samples were taken at the time of disease progression, so that we had insufficient data to examine whether or not ESR1 mutation detection is dependent on specific hormone therapies. The samples used in this study were obtained for biobanking. Therefore, the time from blood draw to spinning, freezing plasma and then thawing may affect the variability of the data.

In conclusion, our study demonstrates the clinical significance of the burden of on-treatment hotspot ESR1 LBD mutations, both in a snapshot and serially in MBC patients in comparison with $P I K 3 C A$ hotspot mutation status, using multiplex ddPCR assays.

\section{MATERIALS AND METHODS}

\section{Patients and breast cancer samples}

A total of 86 patients (185 plasma samples) with breast carcinoma, treated at Kumamoto University Hospital between 2003 and 2016, were enrolled in this study. Cases were selected if archival plasma samples were available. Informed consent was obtained from all patients before biopsy or surgery. The Ethics Committee of Kumamoto University Graduate School of Medicine (Kumamoto, Japan) approved the study protocol. Adjuvant and neoadjuvant treatment was administered in accordance with the recommendations of the St. Gallen international expert consensus on the primary therapy of early BC [29-31]. The treatment of MBC patients was performed in accordance with the National Comprehensive Cancer Network Clinical Practice Guidelines in Oncology [26]. Recurrence was defined as the identification of positive spots by physical examination and/or by imaging diagnosis during the follow-up period. Patients were examined at the Kumamoto University Hospital or affiliated hospitals every 3 months for 5 years and every year thereafter and they were assessed monthly or at longer intervals depending on their disease status.

\section{Sample preparation}

Blood collected in EDTA $\mathrm{K}_{2}$ tubes was processed as soon as possible and was centrifuged at 1,467 $\mathrm{g}$ for 10 min, with plasma stored frozen until DNA extraction. DNA was extracted from $500 \mu \mathrm{L}$ aliquots of plasma using the ISOSPIN Blood \& Plasma DNA kit (Nippon Gene, Tokyo, Japan) according to the manufacturer's instructions. All DNA extracts were quantified using a NanoDrop 2000 spectrometer (NanoDrop Technologies, Wilmington, DE, USA) and purity was determined from the A260/A280 absorbance ratios.

\section{Analysis of ESR1 mutations by ddPCR}

We performed duplicate ddPCR assay on a QX200 digital PCR system (Bio-Rad laboratories, Hercules, CA, USA) using the assays as described previously [17, 32]. The PCR data were quantified as copies/ $\mu \mathrm{L}$ and fractional abundance (allele frequency) using QuantaSoft ${ }^{\mathrm{TM}}$ software (Bio-Rad laboratories). A mutation was considered positive with more than three ESR1 mutant or PIK3CA droplets. The uniplex ddPCR method had been optimized beforehand by comparative analysis of a dilution series of synthetic copies of each indicated mutant ESR1 oligonucleotide, as reported previously [17, 22].

\section{Probes and primers}

We used LBx ${ }^{\circledR}$ Probe ESR1 Multi (A082) as the detection probe for ESR1 Y537S/Y537N, and D538G, $\mathrm{LBx}^{\circledR}$ Probe PIK3CA Screen1 (A087) as the detection probe for PIK3CA E542K/V and E545V/G/A/Q/K, $\mathrm{Q} 546 \mathrm{~L} / \mathrm{R} / \mathrm{P} / \mathrm{E} / \mathrm{K}$, and $\mathrm{LBx}^{\circledR}$ Probe PIK3CA Screen2 (A088) as the detection probe for PIK3CA H1047L/ $\mathrm{R} / \mathrm{Y}$ and G1049R/S (Riken Genesis, Tokyo, Japan), and Custom TaqMan SNP Genotyping assays (Applied Biosystems, Foster City, CA, USA) for the detection of other ESR1 LBD mutations (Y537S, Y537N, and D538G), as described previously [22].

\section{Immunohistochemistry}

Immunohistochemical staining was carried out on $4-\mu \mathrm{m}$ thick tumor sections. Serial sections were prepared from selected blocks and float-mounted on adhesivecoated glass slides for $\mathrm{ER} \alpha, \mathrm{PgR}, \mathrm{HER} 2$, and $\mathrm{Ki} 67$ 
staining. Primary antibodies, their visualization methods, and their evaluation were as previously described [33].

\section{Statistical analysis}

The chi-square test or Fisher's exact test was used to assess baseline differences between binary variables. Correlations were calculated using Spearman's rank correlation coefficient. In the analysis of duration of ET effectiveness, the Kaplan-Meier method was used to estimate survival rates, and differences between survival curves were evaluated by the log-rank test. Cox's proportional hazards model was used for the univariate and multivariate analysis of prognostic status. $P$ values $<$ 0.05 were considered a significant result. All reported $P$ values are two-sided, and CIs are at the 95\% level. All statistical analyses were two-sided and performed using JMP software version 10.0.1 for Windows (SAS institute Japan, Tokyo, Japan).

\section{ACKNOWLEDGMENTS}

This work was supported in part by a grant-in-aid (project numbers 26461952 and 15K10057) for scientific research from the Ministry of Education, Science and Culture of Japan.

\section{CONFLICTS OF INTEREST}

All the authors declare that they have no actual, potential, or perceived conflict of interest with regard to the manuscript submitted for review.

\section{Editorial note}

This paper has been accepted based in part on peerreview conducted by another journal and the authors' response and revisions as well as expedited peer-review in Oncotarget.

\section{REFERENCES}

1. Iwase H. Current topics and perspectives on the use of aromatase inhibitors in the treatment of breast cancer. Breast Cancer. 2008; 15: 278-90. doi: 10.1007/s12282-0080071-y.

2. Li S, Shen D, Shao J, Crowder R, Liu W, Prat A, He X, Liu S, Hoog J, Lu C, Ding L, Griffith OL, Miller C, et al. Endocrine-therapy-resistant ESR1 variants revealed by genomic characterization of breast-cancer-derived xenografts. Cell Rep. 2013; 4: 1116-30. doi: 10.1016/j. celrep.2013.08.022.

3. Toy W, Shen Y, Won H, Green B, Sakr RA, Will M, Li Z, Gala K, Fanning S, King TA, Hudis C, Chen D, Taran T, et al. ESR1 ligand-binding domain mutations in hormoneresistant breast cancer. Nat Genet. 2013; 45: 1439-45. doi: 10.1038/ng.2822.

4. Jeselsohn R, Yelensky R, Buchwalter G, Frampton G, Meric-Bernstam F, Gonzalez-Angulo AM, Ferrer-Lozano J, Perez-Fidalgo JA, Cristofanilli M, Gomez H, Arteaga CL, Giltnane J, Balko JM, et al. Emergence of constitutively active estrogen receptor-alpha mutations in pretreated advanced estrogen receptor-positive breast cancer. Clin Cancer Res. 2014; 20: 1757-67. doi: 10.1158/1078-0432. CCR-13-2332.

5. Karnik PS, Kulkarni S, Liu XP, Budd GT, Bukowski RM. Estrogen receptor mutations in tamoxifen-resistant breast cancer. Cancer Res. 1994; 54: 349-53.

6. Roodi N, Bailey LR, Kao WY, Verrier CS, Yee CJ, Dupont WD, Parl FF. Estrogen receptor gene analysis in estrogen receptor-positive and receptor-negative primary breast cancer. J Natl Cancer Inst. 1995; 87: 446-51.

7. Zhang QX, Borg A, Wolf DM, Oesterreich S, Fuqua SA. An estrogen receptor mutant with strong hormone-independent activity from a metastatic breast cancer. Cancer Res. 1997; 57: 1244-9.

8. Weis KE, Ekena K, Thomas JA, Lazennec G, Katzenellenbogen BS. Constitutively active human estrogen receptors containing amino acid substitutions for tyrosine 537 in the receptor protein. Mol Endocrinol. 1996; 10: 1388-98. doi: 10.1210/mend.10.11.8923465.

9. Diaz LA Jr, Bardelli A. Liquid biopsies: genotyping circulating tumor DNA. J Clin Oncol. 2014; 32: 579-86. doi: 10.1200/JCO.2012.45.2011.

10. Saal LH, Holm K, Maurer M, Memeo L, Su T, Wang X, Yu JS, Malmstrom PO, Mansukhani M, Enoksson J, Hibshoosh H, Borg A, Parsons R. PIK3CA mutations correlate with hormone receptors, node metastasis, and ERBB2, and are mutually exclusive with PTEN loss in human breast carcinoma. Cancer Res. 2005; 65: 2554-9. doi: 10.1158/0008-5472-CAN-04-3913.

11. Stemke-Hale K, Gonzalez-Angulo AM, Lluch A, Neve RM, Kuo WL, Davies M, Carey M, Hu Z, Guan Y, Sahin A, Symmans WF, Pusztai L, Nolden LK, et al. An integrative genomic and proteomic analysis of PIK3CA, PTEN, and AKT mutations in breast cancer. Cancer Res. 2008; 68: 6084-91. doi: 10.1158/0008-5472.CAN-07-6854.

12. Hortobagyi GN, Chen D, Piccart M, Rugo HS, Burris HA 3rd, Pritchard KI, Campone M, Noguchi S, Perez AT, Deleu I, Shtivelband M, Masuda N, Dakhil S, et al. Correlative Analysis of Genetic Alterations and Everolimus Benefit in Hormone Receptor-Positive, Human Epidermal Growth Factor Receptor 2-Negative Advanced Breast Cancer: Results From BOLERO-2. J Clin Oncol. 2016; 34: 419-26. doi: 10.1200/JCO.2014.60.1971.

13. Chandarlapaty S, Chen D, He W, Sung P, Samoila A, You D, Bhatt T, Patel P, Voi M, Gnant M, Hortobagyi G, Baselga J, Moynahan ME. Prevalence of ESR1 Mutations in Cell-Free DNA and Outcomes in Metastatic Breast 
Cancer: A Secondary Analysis of the BOLERO-2 Clinical Trial. JAMA Oncol. 2016; 2: 1310-5. doi: 10.1001/ jamaoncol.2016.1279.

14. Moynahan ME, Chen D, He W, Sung P, Samoila A, You D, Bhatt T, Patel P, Ringeisen F, Hortobagyi GN, Baselga J, Chandarlapaty S. Correlation between PIK3CA mutations in cell-free DNA and everolimus efficacy in HR+, HER2advanced breast cancer: results from BOLERO-2. Br J Cancer. 2017; 116: 726-30. doi: 10.1038/bjc.2017.25.

15. Fribbens C, O'Leary B, Kilburn L, Hrebien S, GarciaMurillas I, Beaney M, Cristofanilli M, Andre F, Loi S, Loibl S, Jiang J, Bartlett CH, Koehler M, et al. Plasma ESR1 Mutations and the Treatment of Estrogen ReceptorPositive Advanced Breast Cancer. J Clin Oncol. 2016; 34: 2961-8. doi: 10.1200/JCO.2016.67.3061.

16. Cristofanilli M, Turner NC, Bondarenko I, Ro J, Im SA, Masuda N, Colleoni M, DeMichele A, Loi S, Verma $\mathrm{S}$, Iwata H, Harbeck N, Zhang K, et al. Fulvestrant plus palbociclib versus fulvestrant plus placebo for treatment of hormone-receptor-positive, HER2-negative metastatic breast cancer that progressed on previous endocrine therapy (PALOMA-3): final analysis of the multicentre, doubleblind, phase 3 randomised controlled trial. Lancet Oncol. 2016; 17: 425-39. doi: 10.1016/S1470-2045(15)00613-0.

17. Takeshita T, Yamamoto Y, Yamamoto-Ibusuki M, Inao T, Sueta A, Fujiwara S, Omoto Y, Iwase H. Clinical significance of monitoring ESR1 mutations in circulating cell-free DNA in estrogen receptor positive breast cancer patients. Oncotarget. 2016; 7:32504-18. doi: 10.18632/ oncotarget.8839.

18. Zonta E, Garlan F, Pecuchet N, Perez-Toralla K, Caen O, Milbury C, Didelot A, Fabre E, Blons H, Laurent-Puig P, Taly V. Multiplex Detection of Rare Mutations by Picoliter Droplet Based Digital PCR: Sensitivity and Specificity Considerations. PLoS One. 2016; 11: e0159094. doi: 10.1371/journal.pone.0159094.

19. Schiavon G, Hrebien S, Garcia-Murillas I, Cutts RJ, Pearson A, Tarazona N, Fenwick K, Kozarewa I, LopezKnowles E, Ribas R, Nerurkar A, Osin P, Chandarlapaty S, et al. Analysis of ESR1 mutation in circulating tumor DNA demonstrates evolution during therapy for metastatic breast cancer. Sci Transl Med. 2015; 7: 313ra182. doi: 10.1126/ scitranslmed.aac 7551.

20. Hrebien S, O’Leary B, Beaney M, Schiavon G, Fribbens C, Bhambra A, Johnson R, Garcia-Murillas I, Turner N. Reproducibility of Digital PCR Assays for Circulating Tumor DNA Analysis in Advanced Breast Cancer. PLoS One. 2016; 11: e0165023. doi: 10.1371/journal. pone.0165023.

21. Toy W, Weir H, Razavi P, Lawson M, Goeppert AU, Mazzola AM, Smith A, Wilson J, Morrow C, Wong WL, De Stanchina E, Carlson KE, Martin TS, et al. Activating ESR1 Mutations Differentially Affect the Efficacy of ER Antagonists. Cancer Discov. 2017; 7: 277-87. doi: 10.1158/2159-8290.CD-15-1523.
22. Takeshita T, Yamamoto Y, Yamamoto-Ibusuki M, Inao T, Sueta A, Fujiwara S, Omoto Y, Iwase H. Droplet digital polymerase chain reaction assay for screening of ESR1 mutations in 325 breast cancer specimens. Transl Res. 2015; 166:540-553.e2. doi: 10.1016/j.trs1.2015.09.003.

23. Lefebvre C, Bachelot T, Filleron T, Pedrero M, Campone M, Soria JC, Massard C, Levy C, Arnedos M, Lacroix-Triki M, Garrabey J, Boursin Y, Deloger M, et al. Mutational Profile of Metastatic Breast Cancers: A Retrospective Analysis. PLoS Med. 2016; 13: e1002201. doi: 10.1371/ journal.pmed.1002201.

24. Spoerke JM, Gendreau S, Walter K, Qiu J, Wilson TR, Savage H, Aimi J, Derynck MK, Chen M, Chan IT, Amler LC, Hampton GM, Johnston S, et al. Heterogeneity and clinical significance of ESR1 mutations in ER-positive metastatic breast cancer patients receiving fulvestrant. Nat Commun. 2016; 7: 11579. doi: 10.1038/ncomms11579.

25. Arthur LM, Turnbull AK, Renshaw L, Keys J, Thomas JS, Wilson TR, Lackner MR, Sims AH, Dixon JM. Changes in PIK3CA mutation status are not associated with recurrence, metastatic disease or progression in endocrine-treated breast cancer. Breast Cancer Res Treat. 2014; 147: 211-9. doi: 10.1007/s10549-014-3080-x.

26. Gradishar WJ, Anderson BO, Blair SL, Burstein HJ, Cyr A, Elias AD, Farrar WB, Forero A, Giordano SH, Goldstein LJ, Hayes DF, Hudis CA, Isakoff SJ, et al. Breast cancer version 3.2014. J Natl Compr Canc Netw. 2014; 12: 542-90.

27. Clatot F, Perdrix A, Augusto L, Beaussire L, Delacour J, Calbrix C, Sefrioui D, Viailly PJ, Bubenheim M, Moldovan C, Alexandru C, Tennevet I, Rigal O, et al. Kinetics, prognostic and predictive values of ESR1 circulating mutations in metastatic breast cancer patients progressing on aromatase inhibitor. Oncotarget. 2016; 7: 74448-59. doi: 10.18632/oncotarget.12950.

28. Nik-Zainal S, Van Loo P, Wedge DC, Alexandrov LB, Greenman CD, Lau KW, Raine K, Jones D, Marshall J, Ramakrishna M, Shlien A, Cooke SL, Hinton J, et al. The life history of 21 breast cancers. Cell. 2012; 149: 994-1007. doi: 10.1016/j.cell.2012.04.023.

29. Goldhirsch A, Wood WC, Gelber RD, Coates AS, Thurlimann B, Senn HJ. Meeting highlights: updated international expert consensus on the primary therapy of early breast cancer. J Clin Oncol. 2003; 21: 3357-65. doi: 10.1200/JCO.2003.04.576.

30. Goldhirsch A, Glick JH, Gelber RD, Coates AS, Thürlimann B, Senn HJ; Panel members. Meeting highlights: international expert consensus on the primary therapy of early breast cancer 2005. Ann Oncol. 2005; 16: 1569-83. doi: 10.1093/annonc/mdi326.

31. Goldhirsch A, Wood WC, Gelber RD, Coates AS, Thürlimann B, Senn HJ; 10th St. Gallen conference. Progress and promise: highlights of the international expert consensus on the primary therapy of early breast cancer 2007. Ann Oncol. 2007; 18: 1133-44. doi: 10.1093/annonc/ mdm271. 
32. Takeshita T, Yamamoto Y, Yamamoto-Ibusuki M, Inao T, Sueta A, Fujiwara S, Omoto Y, Iwase H. Prognostic role of PIK3CA mutations of cell-free DNA in early-stage triple negative breast cancer. Cancer Sci. 2015; 106:1582-9. doi: $10.1111 /$ cas. 12813 .
33. Takeshita T, Omoto Y, Yamamoto-Ibusuki M, Yamamoto $\mathrm{Y}$, Iwase H. Clinical significance of androgen receptor and its phosphorylated form in breast cancer. Endocr Relat Cancer. 2013; 20: L15-21. doi: 10.1530/ERC-13-0317. 